\title{
Superconducting Solenoids for an International Muon Cooling Experiment*
}

\author{
M. A. Green, and J. M. Rey
}

\begin{abstract}
The international muon ionization cooling experiment MICE will consist of two focusing cooling cells and a pair of uniform field solenoids used for particle identification and emittance measurements. The 2.75-meter long cooling cells have a pair of field flip coils and a coupling coil. The 0.52-meter diameter field flip coils surround an absorber that removes transverse and longitudinal momentum from the muons to be cooled. The beam in the absorber is at a minimum beta point so that scattering of the muons is minimized. The 1.7-meter diameter coupling coils are outside of conventional 201.25 MHz RF cavities that accelerate the muons putting longitudinal momentum into the muons without putting back the transverse momentum into the beam. A third set of flip coils helps the muon beam transition from and to the experimental solenoids. The 0.6-meter diameter experimental solenoids have a uniform field region (good to about 1 part in 1000 ) that is 1.3-meters long. The MICE experiment magnets must operate as a single unit so that the field profile will produce the maximum muon cooling.
\end{abstract}

Index Terms-Superconducting Solenoids, and Muon Cooling

\section{. INTRODUCTION}

$\mathrm{T}$ he development of a muon collider or a neutrino factory requires that beams of low emittance muons be produced. A key to the production of low emittance muons is muon cooling. A demonstration of muon cooling is essential to the development of muon accelerators and storage rings. The international Muon Ionization Cooling Experiment (MICE) will be a demonstration of muon cooling in a configuration that may be useful for either a neutrino factory [1] or a muon collider [2].

Ionization cooling of muons means that muons have their momentum reduced in both the longitudinal direction and the transverse direction by passing them through a low $\mathrm{Z}$ absorber. RF cavities are used to re-accelerate the muons to their original momentum. If the scattering in the absorbing medium is not too large, the reaccelerated muon beam will have a lower emittance than the muon beam that entered the absorbers. In order to reduce the multiple scattering of the muon beam in the absorber, the muon beam beta in the absorber must be low and the absorber must have a low $\mathrm{Z}$. The possible absorbers include liquid hydrogen, liquid helium, lithium hydride lithium metal or polyethylene.

Manuscript received 6 August 2002. The M. A. Green is from the Lawrence Berkeley National Laboratory, Berkeley CA 94720 USA. J. M. Rey is from CEN Saclay, 91191 Gif-sur-Yvette, France. *This work was performed at the Lawrence Berkeley Laboratory with the support of the Office of Science at the Department of Energy under DOE contract number DE-AC03-76SF00098.

\section{THE PROPOSED MICE EXPERIMENT}

The proposed MICE experiment will test cooling on a low intensity muon beam from an existing accelerator. It is probable that the MICE will be done at the Rutherford Appleton Laboratory in the United Kingdom. The pions that decay to muons are produced by dipping a target into the ISIS proton beam. The Pions will decay into a well-defined beam of muons. The muon beam will be carried to an experimental hall containing the MICE.

The beam enters the experiment by passing through a foil that will scatter the muon to produce a beam of desired emittance. The muon emittance will be measured by either a time projection chamber (TPC) or four planes of scintillating fibers that are in a uniform solenoidal magnetic field of about $4 \mathrm{~T}$. The required magnetic field uniformity depends on the type of detector selected. Fig. 1 shows a view of MICE.

Once the emittance of the muon beam entering the cooling section has been measured, the beam passes through an absorber that cools the muon beam by ionization cooling. The absorber is in a field flip region (where the direction of the field reverses) so that the beam beta can be minimized. The absorber may be liquid hydrogen, liquid helium, lithium hydride, lithium, or plastic. The preferred absorber material is liquid hydrogen, because it has the lowest multiple scattering of any of the absorber materials for a given energy absorption and beam beta. Comparable performance from lithium or lithium hydride requires a cooling lattice that produces the lowest possible beam beta. The use of liquid hydrogen absorbers presents the design team with safety issues, with which many sites do not want to deal.

The muon beam momentum is recovered by accelerating the beam with a four cell $201.25 \mathrm{MHz}$ RF cavity that is in a 2.5 to $3 \mathrm{~T}$ magnetic field. After the muon beam has been reaccelerated, it passes through a second absorber in a second field flip region. The process of re-acceleration is repeated then the beam passes through the final absorber and field flip solenoid. At this point, the muon beam should be cooled so that the cooling can be clearly measured. The MICE cooling cells are almost identical to the $2.75-\mathrm{m}$ long muon cooling cells in the level 2 study of the neutrino factory [3] [4].

Once the beam has passed through the ionization cooling section, it enters a second detector section. The second detector section is identical to the first detector, except for the time of flight detectors at the end of the experiment. A critical problem for the MICE experiment is matching between the detectors and the muon cooling section. 


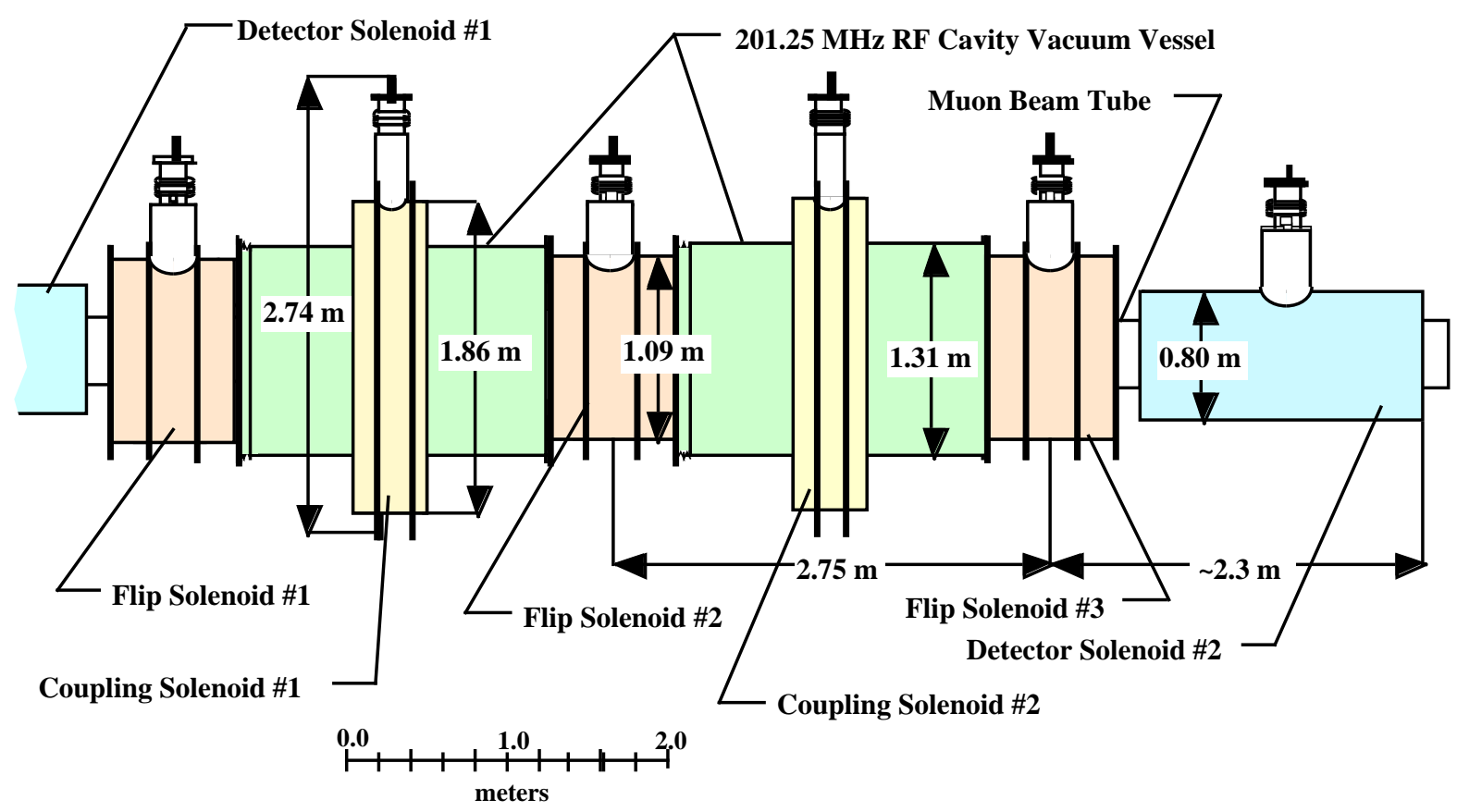

Fig. 1. A Schematic Representation of the MICE Experiment Magnets as Seen from the Outside. The liquid hydrogen absorbers are inside of the flip solenoid cryostats. The $201 \mathrm{MHz}$ RF cavities are inside the cavity vacuum vessel and the coupling magnet.

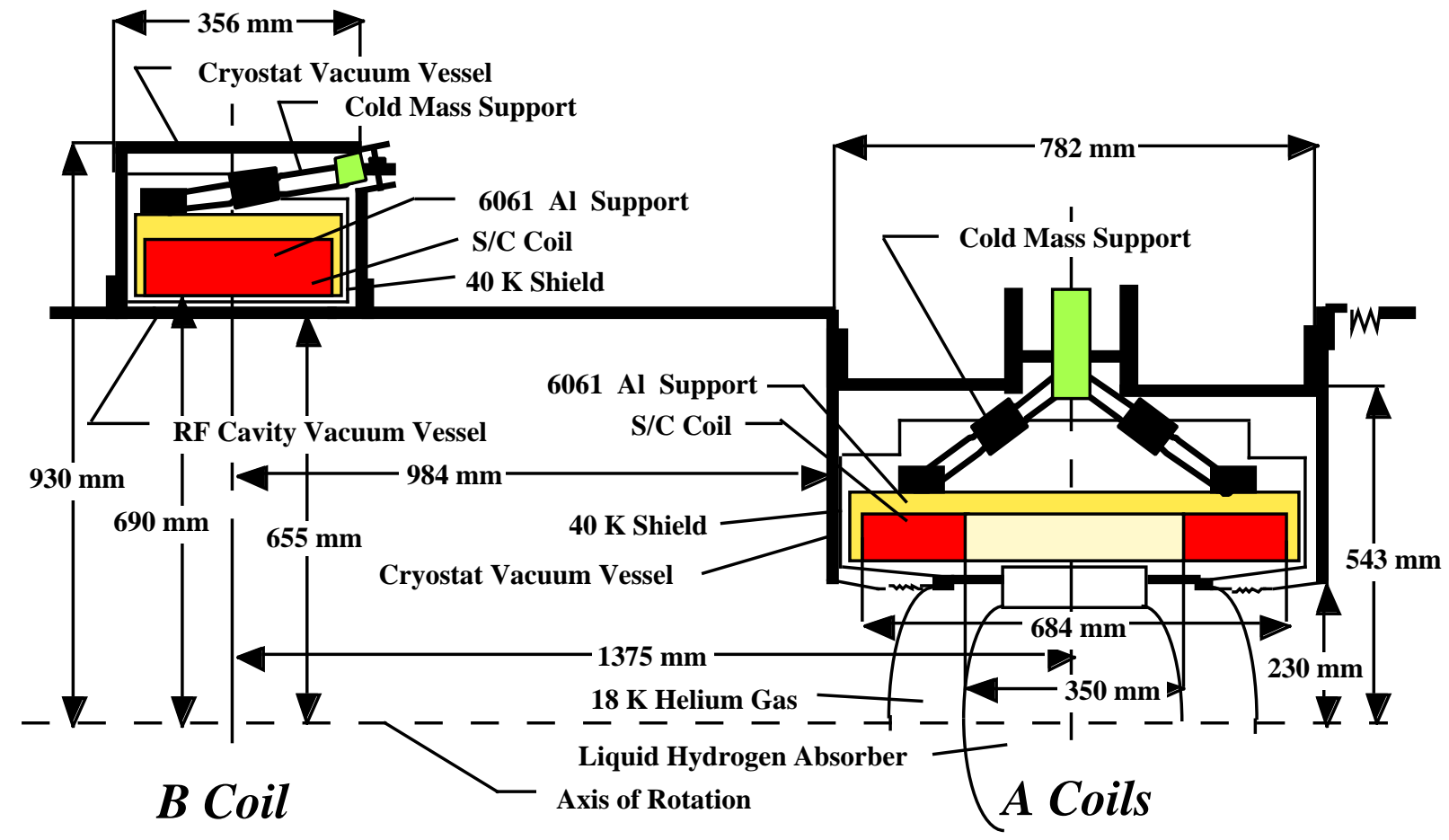

Fig. 2. A Quarter Section of the Superconducting Solenoid Magnets in a Muon Cooling Cell Used in the MICE Experiment.

\section{S UPERCONDUCTING SOLENOIDS FOR MICE}

The muon cooling experiment MICE has superconducting solenoids of three different types: 1) The coupling solenoids create a field in the cavities so that the beam can pass through the MICE without loss. The coupling solenoid surrounds the $201 \mathrm{MHz}$ RF cavities. 2) The field-flip solenoids produce the magnetic field flip in the absorber in order to minimize the beam beta in the absorber. A small beam beta reduces the effects of absorber multiple scattering. 3) The detectors require a relatively uniform field. This uniform field must extend over a distance of 1 meter. Scintillating fiber detectors require a field uniformity of 1 percent. TPC detectors need a field that is uniform to 0.1 percent. 


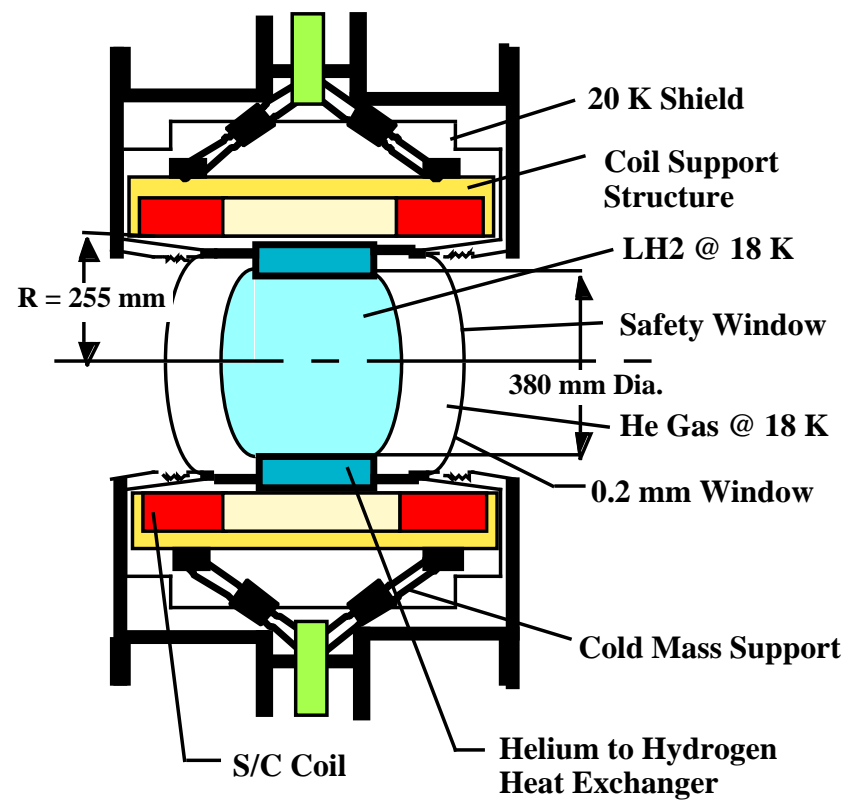

Fig. 3. A schematic representation of a liquid hydrogen absorber integrated with the flip solenoid. Note: The liquid hydrogen absorber has an additional safety window. Helium gas at $18 \mathrm{~K}$ will be in the space between the liquid hydrogen absorber window and the safety window. The field along the axis of the flip solenoid varies from $3.5 \mathrm{~T}$ to $-3.5 \mathrm{~T}$. The field at the center of the coil pair is zero.

The cost has been issue for MICE. Primary costs are in the detectors, the RF system, and the solenoids. The cooling cell shown in Fig. 2 is nearly identical to the long cooling cell proposed in the level 2 study [5]. The stored energy for each level 2 study cooling-cell was 13 MJ. In order to reduce the cost of the solenoid magnets, they had to be made smaller. Since the size of the coupling solenoid is dictated by the diameter of the RF cavity, only modest changes in the coil diameter could be made. The level 2 study flip solenoid diameter could be reduced provided one is willing to integrate the solenoid coils with the absorber. Integration of the flip solenoid allowed one to reduce the solenoid ID from $700 \mathrm{~mm}$ to $500 \mathrm{~mm}$. Fig. 3 shows how the reduced size flip solenoid pair might be integrated with a liquid hydrogen absorber with 380-mm diameter thin windows.

Reduction of the flip coil ID produced a number of dividends: 1) The current in the flip coils needed to produce the field flip was reduced a factor of two, which led to a reduction of coil thickness. 2) The magnetic force pushing the flip coils apart was reduced by a factor four. This has a profound effect on the coil support structure. 3) The force between the flip coils and the coupling coils during a magnet quench is reduced over a factor of two. This allows one to use a conventional cold mass support system for both the flip solenoid and the coupling solenoids. 4) The stored energy of the flip solenoid pair was reduced over a factor of six. 5) The beam beta in the absorber was reduced over the full range of muon momenta. In a neutrino factory this means that the ionization cooling will be more effective over a longer length. 6) The cost of the flip solenoid is reduced a factor of two despite the additional complication on the magnet design imposed by the requirement that the flip solenoid be integrated with the absorber system.

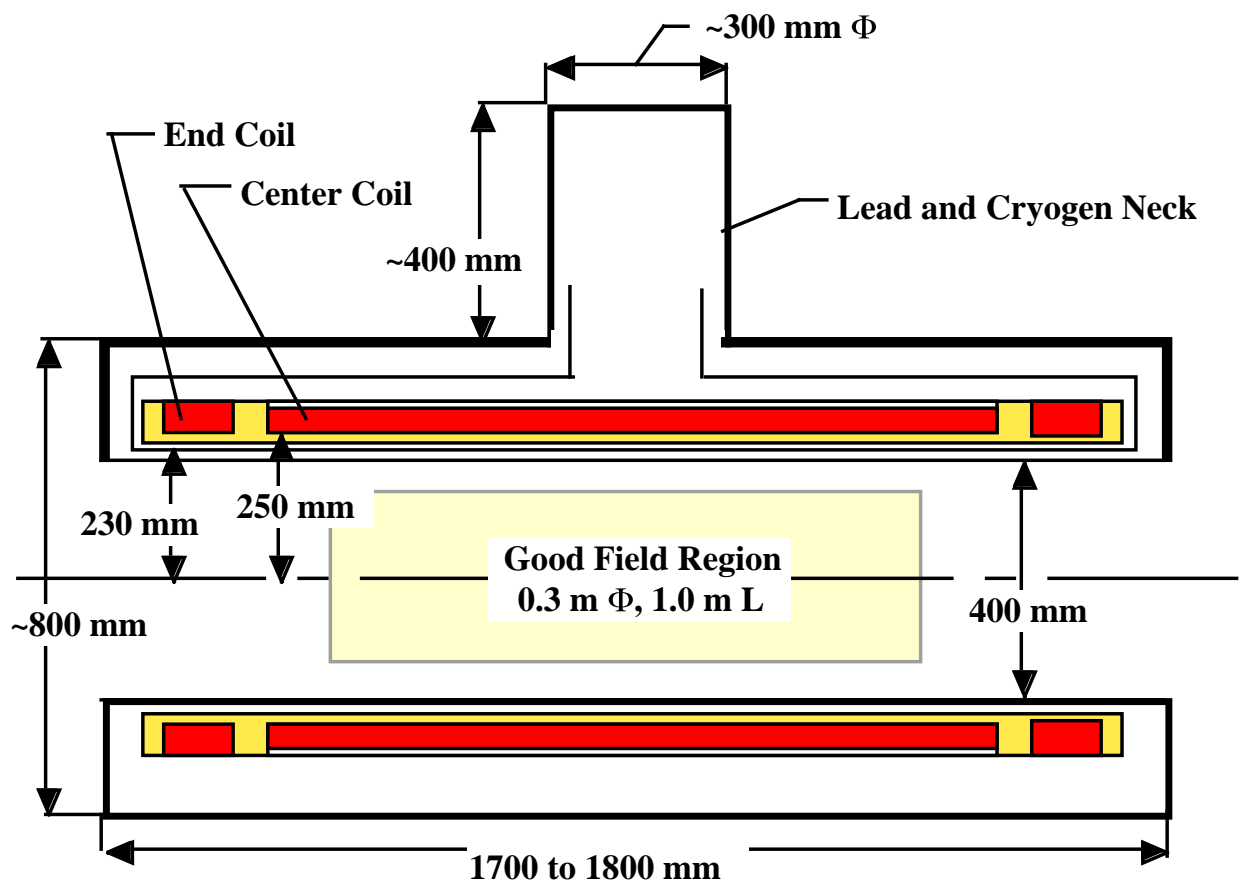

Fig. 4. A Cross-section Schematic View of a 4 T MICE Detector Solenoid that can Produce a Uniform Magnetic Field 1-meter Long. The magnet shown will produce a field uniformity of 1 percent over the good field region. Additional coils may be needed to produce a field uniformity of 0.1 percent in the good field region. Leads and cryogenic services are not shown in the cryostat service neck. 
A schematic cross-section of a MICE detector solenoid is shown in Fig. 4. The detector solenoid is shown as a classic three-coil solenoid that produces a uniform field. The solenoid shown in Fig. 4 should be capable of producing a uniform field in the designated good field region of $300 \mathrm{~mm}$ in diameter and $1000 \mathrm{~mm}$ long. As a stand alone magnet, a three coil solenoid can be designed to produce a field uniformity of 0.1 percent in the good field region. The detector solenoid does not stand alone. It is part of a system of superconducting solenoids. As a result, the detector solenoid may need additional coils to shim the field in the good field region to the 0.1 percent uniformity level while it is operating in the presence of other solenoids.

Table I summarizes the coil parameters of the three types of solenoids for MICE. The values given in Table I represent the nominal design values for the MICE solenoids. The final design of the solenoid may be governed by tests of cooling of off momentum muons. An increase in the average muon momentum from $200 \mathrm{MeV} / \mathrm{c}$ to say $240 \mathrm{MeV} / \mathrm{c}$ may affect the design current for the solenoids in the cooling channel. The detector solenoids may or may not be affected by changing the central momentum of the muons. Further simulation of Muon cooling is needed to determine the design range for the MICE solenoids.

TABLE 1

NOMINAL DESIGN PARAMETERS FOR MICE SOLENOIDS

\begin{tabular}{lccc} 
Component & $\begin{array}{c}\text { Coupling } \\
\text { Solenoid }\end{array}$ & $\begin{array}{c}\text { Flip } \\
\text { Solenoid }\end{array}$ & $\begin{array}{c}\text { Detector } \\
\text { Solenoid }\end{array}$ \\
\hline Warm Bore Diameter (mm) & 1310 & 460 & 400 \\
Warm Outer Diameter (mm) & 1860 & 1090 & $\sim 800$ \\
Cryostat Length (mm) & 380 & 780 & $\sim 1700$ \\
Coil Inner Diameter (mm) & 1380 & 510 & 500 \\
Coil Thickness (mm) & 90 & 75 & 32 \\
Coil Length (mm) & 280 & 167 & 1500 \\
Coil Spacing & --- & 350 & $\sim 60$ \\
Central Induction (T) & $\sim 2.8$ & 0 & 4.0 \\
Magnetic Gradient (T/m) & --- & $\sim 20$ & --- \\
Design Coil J (A mm $\left.{ }^{-2}\right)$ & $\sim 90$ & $\sim 128$ & $\sim 100$ \\
Design S/C Matrix J (A mm $\left.{ }^{-2}\right)$ & 138 & 182 & 150 \\
Stored Energy (MJ) & $\sim 5.5$ & $\sim 0.6$ & $\sim 3.5$ \\
Overall Mass (kg) & 1350 & 350 & 1350
\end{tabular}

\section{INTERACTIONS BETWEEN MICE SOLENOIDS}

The solenoids in the MICE are not alone. The MICE solenoid design does not call for any of the solenoids to be shielded. Since there are three field flips in MICE (one field flip in one configuration of the experiment), the net magnetic moment for all of the magnets is zero. At $10-\mathrm{m}$ from the MICE beam axis, the field will fall to the $0.0001 \mathrm{~T}$ level. During initial stages of the experiment, the net magnetic moment may not be zero. The stray field will be higher at this time. The implications of the MICE solenoid stray field will have to be better understood.
Even when the MICE operates in its nominal mode, there will be longitudinal forces between the solenoid coils. During a worse case fault (when one coil quenches and the others do not), the magnitude of the longitudinal magnetic force is about 35 metric tons. The implication of this is that the cold mass supports for the coupling coils and the flip coils must be designed to carry this force during a fault. The detector solenoids and the outer flip solenoids will never see a balanced force during normal operation. The longitudinal forces that occur during normal operation of MICE will be lower than the fault forces.

During the proposed mode of MICE operation, it is proposed that both coupling coils will be powered with a single power supply. The three flip coil solenoid pairs can also be powered from a common power supply. The detector solenoids may be powered separately or together.

Each of the flip solenoid coils should have a set of leads so that each coil in the pair can be powered separately. This allows for maximum flexibility in the experiment. It is proposed that each coil (for a total 14) have its own trickle power supply so that the current in each coil can be varied independently by \pm 10 percent of the nominal current for that coil. This will allow one to tune the MICE magnets to maximize cooling and muon throughput.

\section{CONCLUding COMMENTS}

The superconducting solenoids for MICE can be built with existing niobium titanium conductors. Integrating the flip solenoids with the absorber system will result in better muon cooling performance and lower cost magnets. The coupling solenoid cost can also be reduced, by moving the coil current center closer to the $201 \mathrm{MHz}$ RF cavities. The detector solenoids must be combined with the rest of the magnets in the MICE cooling channel. The end coils of the detector solenoid and the coils in the flip solenoids must be separately tunable so that the muon beam can be properly tuned to maximize cooling and throughput through the magnet system over a range of momenta.

\section{REFERENCES}

[1] N. Holtkamp and D. Finley Eds., "A Feasibility Study of a Neutrino Source Based on a Muon Storage Ring," FERMI-Pub-00/108E, (2000)

[2] R. B. Palmer, A. Sessler, A. Skrinsky, A. Tollestrup, et al, "Muon Colliders, "Brookhaven National Laboratory Report BNL-62740, January 1996

[3] S. Ozaki, R. B. Palmer, M. S. Zisman and J. Gallardo eds. "Feasibility Study II of a Muon Based Neutrino Source," BNL-52623, June 2001

[4] M. S. Zisman, "Status of Neutrino Factory and Muon Collider R and D," US Particle Accelerator Conference Proceedings, (2001)

[5] M. A. Green, J. R. Miller, R. B. Palmer, et al, "Solenoid Magnets for the Front End of a Neutrino Factory," proceedings of the Snowmass Summer Study on Issues in Particle Physics, (2001) 


\section{DISCLAIMER}

This document was prepared as an account of work sponsored by the United States Government.

While this document is believed to contain correct information, neither the United States Government nor any agency thereof, nor The Regents of the University of California, nor any of their employees, makes any warranty, express or implied, or assumes any legal responsibility for the accuracy, completeness, or usefulness of any information, apparatus, product, or process disclosed, or represents that its use would not infringe privately owned rights. Reference herein to any specific commercial product, process, or service by its trade name, trademark, manufacturer, or otherwise, does not necessarily constitute or imply its endorsement, recommendation, or favoring by the United States Government or any agency thereof, or The Regents of the University of California. The views and opinions of authors expressed herein do not necessarily state or reflect those of the United States Government or any agency thereof, or The Regents of the University of California.

Ernest Orlando Lawrence Berkeley National Laboratory is an equal opportunity employer. 